\title{
An evaluation of three processing methods and the effect of reduced culture times for faster direct identification of pathogens from BacT/ALERT blood cultures by MALDI-TOF MS
}

\author{
A. J. M. Loonen • A. R. Jansz • J. Stalpers • \\ P. F. G. Wolffs • A. J. C. van den Brule
}

Received: 31 August 2011 / Accepted: 24 October 2011 /Published online: 12 November 2011

(C) The Author(s) 2011. This article is published with open access at Springerlink.com

\begin{abstract}
Matrix-assisted laser desorption/ionisation timeof-flight mass spectrometry (MALDI-TOF MS) is a fast and reliable method for the identification of bacteria from agar media. Direct identification from positive blood cultures should decrease the time to obtaining the result. In this study, three different processing methods for the rapid direct identification of bacteria from positive blood culture bottles were compared. In total, 101 positive aerobe BacT/ALERT bottles were included in this study. Aliquots from all bottles were used for three bacterial processing methods, i.e. the commercially available Bruker's MALDI Sepsityper kit, the commercially available Molzym's MolYsis Basic5 kit and a centrifugation/washing method. In addition, the best method was used to evaluate the possibility of MALDI application after a reduced incubation time of $7 \mathrm{~h}$ of Staphylococcus aureus- and Escherichia coli-spiked (1,000, 100 and 10 colony-forming units
\end{abstract}

A. J. M. Loonen · A. R. Jansz · J. Stalpers · A. J. C. van den Brule Department of Medical Microbiology, PAMM Laboratories,

Veldhoven/Eindhoven, The Netherlands

\author{
A. J. M. Loonen • A. J. C. van den Brule \\ Department of Medical Molecular Diagnostics, \\ Fontys University of Applied Science, \\ Eindhoven, The Netherlands \\ A. J. M. Loonen • P. F. G. Wolffs \\ Department of Medical Microbiology, \\ Maastricht University Medical Centre, CAPHRI, \\ Maastricht, The Netherlands
}

\author{
A. J. M. Loonen · A. J. C. van den Brule $(\bowtie)$ \\ Laboratory for Molecular Diagnostics, Departments of Medical \\ Microbiology and Pathology, Jeroen Bosch Hospital, \\ P.O. Box 90153, $5200 \mathrm{ME}$, 's-Hertogenbosch, The Netherlands \\ e-mail: a.v.d.brule@jbz.nl
}

[CFU]) aerobe BacT/ALERT blood cultures. Sixty-six $(65 \%), 51(50.5 \%)$ and $79(78 \%)$ bottles were identified correctly at the species level when the centrifugation/ washing method, MolYsis Basic 5 and Sepsityper were used, respectively. Incorrect identification was obtained in $35(35 \%), 50(49.5 \%)$ and $22(22 \%)$ bottles, respectively. Gram-positive cocci were correctly identified in 33/52 $(64 \%)$ of the cases. However, Gram-negative rods showed a correct identification in 45/47 (96\%) of all bottles when the Sepsityper kit was used. Seven hours of pre-incubation of $S$. aureus- and E. coli-spiked aerobe BacT/ALERT blood cultures never resulted in reliable identification with MALDI-TOF MS. Sepsityper is superior for the direct identification of microorganisms from aerobe BacT/ALERT bottles. Gram-negative pathogens show better results compared to Gram-positive bacteria. Reduced incubation followed by MALDI-TOF MS did not result in faster reliable identification.

\section{Introduction}

Fast and accurate diagnostic methods are necessary in order to be able to initiate correct treatment for bloodstream infections. Adequate and fast antibiotic treatment reduces the mortality rates in these patients [1, 2]. Cultivation techniques take at least a day, and although the subsequent Gram staining can be performed in several minutes, the result from this staining technique alone does often not provide sufficient information to administer appropriate antibiotic therapy. Therefore, additional tests have to be performed so as to identify the pathogen that causes the disease, leading to an increased time to initiate appropriate therapy. 
Improved techniques have been developed to reduce the time-to-result, i.e. VITEK 2, FISH (fluorescence in situ hybridisation) and (commercial) real-time polymerase chain reaction (PCR)-based methods [3-6]. However, most of these techniques are limited by high costs and the fact that not every species can be detected in one assay. This makes it difficult to implement the technique in routine diagnostics.

Recently, a new method became available in several clinical microbiology laboratories, i.e. matrix-assisted laser desorption/ionisation time-of-flight mass spectrometry (MALDI-TOF MS). This technique can be used for pathogen identification from agar media [7-9]. However, several reports are currently available which describe the possibility of direct identification from positive blood cultures [10-15]. Most reports describe the use of BACTEC bottles, but a few studies $[16,17]$ used BacT/ALERT bottles. This makes the BacT/ALERT system underrepresented in the literature, while it is a frequently used culture system in many laboratories. Pathogens from blood cultures need to be separated from the liquid culture media prior to detection by MALDI-TOF MS. Several similar protocols have been published consisting either of the removal of erythrocytes by centrifugation [11, 12] or serum separation tubes [10,14], followed by subsequently inactivating the bacteria by using ethanol, protein precipitation and extraction by formic acid and acetonitrile.

Recently, new processing methods have become available. Sepsityper (Bruker Daltonics) allows the easy and fast identification of bacteria from positive blood cultures by using MALDI-TOF MS. Another commercially available method, MolYsis (Molzym), is capable of removing human background (cells and DNA) from blood (culture) samples by selective lysis and, additionally, ensures pathogen enrichment. This method has been shown to be suitable for molecular diagnostics [4, 18]. However, it is still unknown as to whether this enrichment method is also feasible for direct pathogen identification from positive blood cultures by MALDI-TOF MS.

In addition, it was demonstrated that accelerated detection of staphylococci from spiked blood cultures was possible after only $5 \mathrm{~h}$ of pre-incubation when, subsequently, MolYsis enrichment was combined with real-time PCR [4].

In the present study, we compared three easy-to-perform protocols, i.e. commercially available MolYsis Basic 5 and Sepsityper, and a non-commercial centrifugation/washing method as described by Ferreira et al. [11], for the direct identification of bacteria from positive aerobe BacT/ ALERT blood cultures with MALDI-TOF MS. The results were compared to conventional diagnostics and MALDITOF MS directly from blood agar. To evaluate the possibility of reduced time-to-identification from blood cultures when using MALDI-TOF MS analysis, the best processing method was also investigated in relation to the reduced incubation times $(7 \mathrm{~h})$ of Staphylococcus aureus- and Escherichia coli-spiked BacT/ALERT blood cultures.

\section{Materials and methods}

\section{Clinical samples}

Blood samples $(8-10 \mathrm{ml})$ from patients were collected in standard aerobe (SA) and anaerobe (SN) BacT/ALERT blood culture bottles (bioMérieux, Marcy l'Etoile, France) without charcoal, and handled according to the manufacturer's instructions. A total of 101 positive aerobe blood cultures (equal distribution of Gram-negative rods and Gram-positive cocci) were collected for this study (see Table 1 for details).

When patient samples were detected as positive by the BacT/ALERT 3D system (bioMérieux, Marcy l'Etoile, France), Gram staining was performed and, based on the Gram result, additional subculture and biochemical tests were performed (conventional diagnostics as schematically shown in Fig. 3a). When Gram-negative rods were detected by Gram staining, several agar media were inoculated for subculture. One additional blood plate was inoculated for a spot-subculture (concentrated spot with blood culture material in the centre of the plate), which was used for the MALDI-TOF MS analysis. Resistance was determined by the use of VITEK 2 (bioMérieux). When Gram-positive cocci were detected in the blood culture sample, several agar media were inoculated for subculture. Standard biochemical tests were performed (based on the Gram result), because MALDI-TOF MS was still being validated in the laboratory for Gram-positive cultures, to obtain identification of the pathogen (coagulase, TLM plate, BAGG test, tellurite plate, rapid strep test, VITEK 2 [CNS species]). As Streptococcus pneumoniae can die in the culture bottle (self-lysing ability), an LytA real-time PCR [19] was performed when no subculture on blood agar was detected. Resistance determination occurred by the use of the disk diffusion method.

One colony of the subculture on blood agar was used for MALDI-TOF MS.

The Internal Review Board of the hospital ethically approves the anonymous use of remaining patient material.

Strains for spiking experiments

The blood of healthy human volunteers $(10 \mathrm{ml})$ was collected in SA BacT/ALERT culture bottles. For spiking experiments, S. aureus (ATCC 25923) and E. coli (ATCC 25922) were used. 
Table 1 Representation of results obtained with conventional culture techniques, MALDI-TOF MS from blood agar and directly from positive blood cultures with different methods

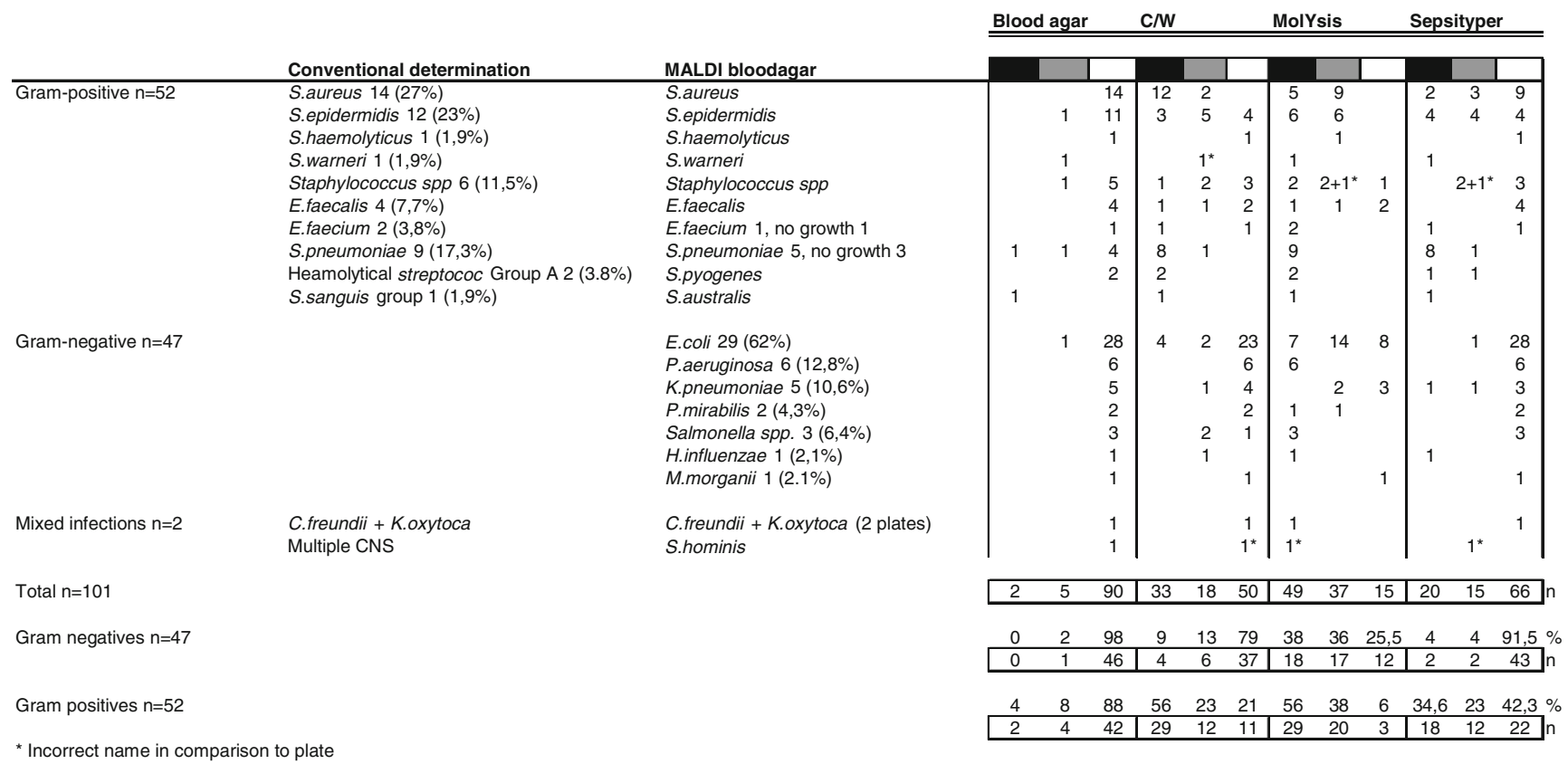

Black colour represents a score $<1.7$, grey colour represents scores $\geq 1.7 \times<2$, and white stands for scores $\geq 2$. C/W; centrifugation/washing method. When "no reliable identification" or "no peaks found" was obtained as a result, the manual selection of areas on the spot of the polished steel target plate was performed to possibly obtain reliable results

\section{Processing methods for MALDI-TOF MS}

From each positive culture bottle, three aliquots were taken; for clarification, see Fig. 1. For the Sepsityper method (Bruker Daltonics GmbH, Bremen, Germany), the recommended quantity of $1 \mathrm{ml}$ was used. The protocol was performed as recommended by the manufacturer. For the MolYsis Basic method (Molzym GmbH, Bremen, Germany), $1 \mathrm{ml}$ was used and the provided protocol was followed until the first washing step. For the third method, $4 \mathrm{ml}$ of positive culture material was used. The centrifugation/washing method was performed as described by Ferreira et al. [11]. All methods resulted in a pellet, which was subsequently dissolved in

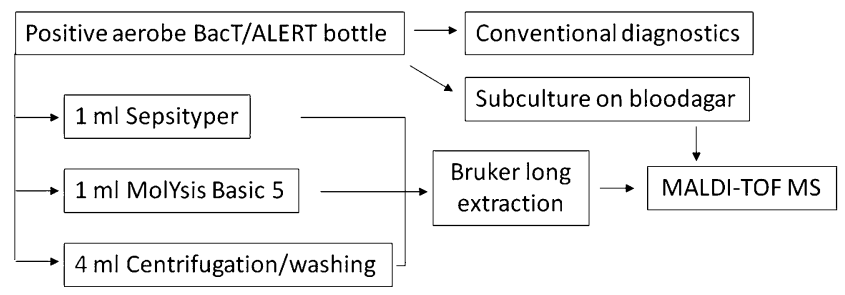

Fig. 1 Overview of bacterial identification from positive aerobe BacT/ALERT cultures by using MALDI-TOF MS. Three aliquots were taken from each positive blood culture bottle to analyse the different processing methods. All methods were performed as described in the Materials and methods section. All results were compared to conventional diagnostics and/or MALDI-TOF MS directly from blood agar
$300 \mu \mathrm{l}$ of water, to which $900 \mu \mathrm{l}$ of ethanol absolute was added. The mixture was centrifuged at 14,000 rpm for $2 \mathrm{~min}$, and the supernatant was discarded. The centrifugation step was repeated so as to remove all residual supernatant. The pellet size was estimated in $\mu \mathrm{l}$ and, to this pellet, in a 1:1 ratio, $70 \%$ formic acid was added and mixed thoroughly. Next, the same amount of acetonitrile was added (1:1 ratio with formic acid) and mixed well before centrifugation at $14,000 \mathrm{rpm}$ for $2 \mathrm{~min}$. One $\mu \mathrm{l}$ of the supernatant was applied on a spot of the polished steel target plate and air-dried at room temperature. The dried spot was overlaid with $1 \mu$ of HCCA matrix (Bruker Daltonics GmbH, Bremen, Germany) solution (saturated alpha-cyano-4-hydroxycinnamic acid in $50 \%$ acetonitrile and $2.5 \%$ trifluoroacetic acid) and allowed to air-dry at room temperature.

\section{MALDI-TOF MS analysis}

Samples were placed in the MALDI-TOF MS spectrometer (BioTyper, Bruker Daltonics GmbH, Bremen, Germany). The spectrometer was calibrated with Bruker bacterial test standard (Bruker Daltonics GmbH, Bremen, Germany). For pathogen identification, 240 shots in 40-shot steps from different positions of the target spot (automatic) were recorded to obtain a spectrum per strain and spectra were imported in the MALDI BioTyper 2.0 software (Bruker Daltonics $\mathrm{GmbH}$, Bremen, Germany) and analysed by 
standard pattern matching; the BioTyper database version V3 1.1.0 3476-3740 was used as a reference. No intervention took place in the process from measurement to identification. For each strain, the highest score obtained by pattern matching to the database was used for the identification. Scores $<1.7$ were considered to unreliable, scores $\geq 1.7 \times<2$ were considered to be reliable to the genus level; however, if database match number 1 and 2 resulted in the same strain name, it was considered correct to the species level, and scores $\geq 2$ were considered to be reliable to the species level. When "no reliable identification" or "no peaks found" was obtained as a result, the manual selection of areas on the spot of the polished steel target plate was performed to possibly obtain reliable results. All data were compared to conventional diagnostics and MALDI-TOF MS results obtained from a colony grown on blood agar.

\section{Statistical analysis}

For analysis of the results, the McNemar test was used. The total amount of correct identifications (scores $\geq 1.7$ with hit 1 and 2 being similar, and scores $>2$ ) and incorrect identifications were compared for all of the methods investigated. A binomial distribution was used.

\section{Results}

One hundred and one aerobe blood cultures detected as positive by the BacT/ALERT 3D system were processed by conventional methods, tested by MALDI-TOF MS from blood agar (direct smear method) and tested directly from positive blood cultures by using three different bacterial isolation methods (Fig. 1). Only aerobe BacT/ALERT bottles were used because, in pilot experiments (preliminary data were obtained with small sample numbers), anaerobe BacT/ALERT bottles frequently resulted in unreliable data. Fifty-two cultures showed the presence of Gram-positive bacteria after Gram staining, 47 cultures showed the presence of Gram-negative bacteria and two cultures indicated, after conventional diagnostics, the presence of multiple bacterial species (Table 1). The direct smear method (blood agar) is the standard diagnostic method for Gram-negative bacteria and resulted in reliable identification. For the Gram-positive cultures, both conventional diagnostics and the direct smear method were performed (due to the ongoing process validation of MALDI-TOF MS diagnostics). Four of 52 blood agar plates did not result in growth. Of these four, three were characterised as $S$. pneumoniae by PCR and one was plated for a second time and was identified as Enterococcus faecium by conventional diagnostics. S. sanguis was detected as $S$. australis by
MALDI-TOF MS from blood agar. Blood culture samples that resulted in "unreliable data" or "no peaks found" were retested immediately from the same spot by the manual selection of areas on the polished steel target plate. This procedure never resulted in acceptable results $(\geq 1.7)$ for further analysis.

Of the methods used for direct identification from positive blood cultures, Sepsityper showed (Table 1 and Fig. 2) a correct identification in $79 / 101$ cultures $(\geq 1.7)$ equal to $78 \%$, in contrast to MolYsis and the centrifugation/ washing method, where only $50.5 \%$ and $65 \%$ were correctly identified, respectively. However, when Gramnegative cultures were separated from Gram-positive cultures and mixed cultures, it can be seen that cultures containing Gram-negatives show more correct identifications with all of the methods used. When Sepsityper was used, 96\% (45/47) of all Gram-negative cultures showed correct identification $(\geq 1.7)$, on the contrary to cultures containing Gram-positives, where only $64 \%$ of the results (33/52) were correct. For the centrifugation method, $92 \%$ (43/47) and for the MolYsis method, 62\% (29/47) of the Gram-negative cultures were identified correctly $(\geq 1.7)$. With both the centrifugation and the MolYsis methods, $42 \%(22 / 52)$ of the Gram-positive cultures were correctly identified.

Staphylococci were most frequently detected in the cultures containing Gram-positive bacteria, i.e. they represented $65 \%$ of these bottles $(34 / 52)$. The direct smear method always resulted in reliable identification by MALDI-TOF MS. When identification directly from the positive blood culture was performed, Sepsityper resulted in the most correct identifications, as 12/14 S. aureus, 8/12 S. epidermidis, 1/1 S. haemolyticus, 0/1 S. warneri and 5/6 Staphylococcus spp. showed identical results compared to the results obtained from the blood agar plate. In this study, streptococci were found to be the second most detected group of bacteria from culture bottles, with 12/52 (23\%) cases. S. pneumoniae was recovered most frequently, in $9 /$ 12 cases. In three cases, no growth was detected on blood agar and, therefore, the direct smear method could not be performed. In five cases, $S$. pneumoniae was identified correctly from blood agar $(\geq 1.7)$; however, directly from positive cultures successful detection of this pathogen proved to be difficult, as correct identification only occurred once with both the centrifugation/washing method and the Sepsityper method $(\geq 1.7 \times<2)$. Enterococci were recovered from $6 / 52$ cultures $(11.5 \%)$; in four cases $E$. faecalis was found and in two cases, E. faecium was found. Enterococci were detected accurately $(>2)$ from blood agar (Table 1), except for one E. faecium strain which did not result in proper growth. Sepsityper characterised all E. faecalis cultures and one E. faecium culture accurately $(>2)$. 
A

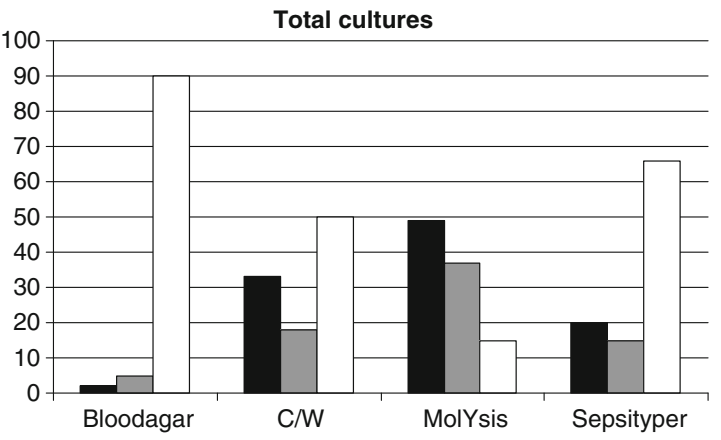

B

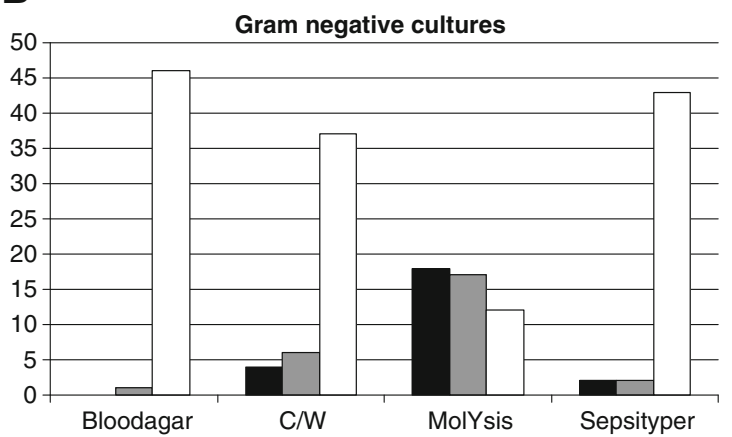

Fig. 2 Overview of results obtained with different bacterial isolation methods from positive blood cultures in comparison to results obtained with the direct smear method directly from blood agar. a The overall results are depicted obtained after the analysis of all bottles included in this study. b Specific results obtained from the 47

The McNemar test was used to investigate the statistical significance of the obtained results. From the analysis of the total amount of blood cultures tested, it was shown that the direct smear method always showed significantly better results compared to identification directly from positive blood cultures (all methods), $p \leq 0.001$. The Sepsityper method showed to lead to significantly better results compared to the MolYsis enrichment, $p=0.000$. No significant difference was observed between the $\mathrm{C} / \mathrm{W}$ method and MolYsis $(p=0.11)$ and the $\mathrm{C} / \mathrm{W}$ method and Sepsityper $(p=0.19)$. When only the blood cultures containing Gram-positive bacteria were analysed, by using the McNemar test, it was again shown that the direct smear method was always significantly better compared to analysis directly from blood cultures, $p \leq 0.002$. Sepsityper proved to be significantly better compared to the MolYsis treatment, $p=0.008$. The $\mathrm{C} / \mathrm{W}$ method was comparable to Sepsityper and MolYsis. As the direct smear method for all Gram-negative-containing blood cultures always resulted in correct identification, these results were not used for McNemar analysis. The $\mathrm{C} / \mathrm{W}$ method and the Sepsityper method performed on positive blood cultures (Gram-negative bacteria) lead to significantly better results compared to MolYsis $(p \leq 0.01)$, but not when compared to each other.
$<1.7$

$\geq 1.7 x<2.0$

$\geq 2.0$

C

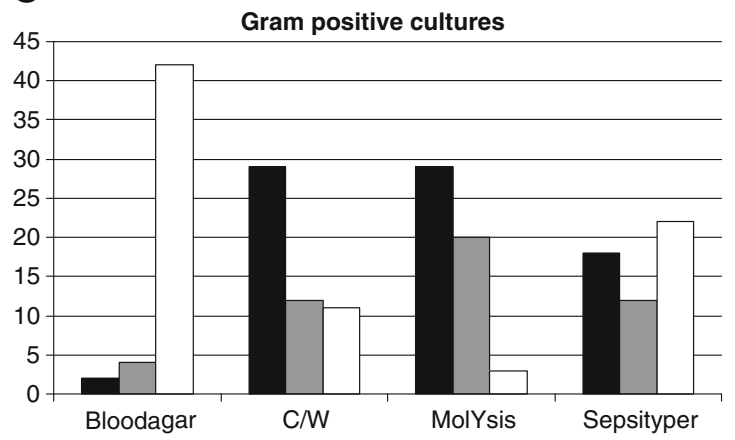

bottles containing Gram-negative bacteria. $\mathbf{c}$ The data of the 52 bottles in which Gram-positive bacteria were found are shown. C/W; centrifugation/washing method. Statistical significance was analysed by using the McNemar test, see the Results section for details

The time needed to process positive cultures with the described methods varied. Sepsityper was fast $(20 \mathrm{~min})$ and easy to use, while MolYsis (50 $\mathrm{min}$ ) had quite some incubation steps, took a lot of hands-on time and did not lead to reliable results. The centrifugation method is a good alternative (30 $\mathrm{min}$ ), especially for Gram-negative pathogens, as it is cheap to use and shows promising results for this group of micro-organisms. In contrast, the commercially available methods are more expensive (the list price in The Netherlands for the Sepsityper and Molzym methods are 4 and 8 Euros per test, respectively).

In preliminary experiments, BacT/ALERT bottles with charcoal were also analysed by using the Sepsityper method (Bruker) and the method described by Moussaoui et al. [14], using serum separation tubes. Bottles containing charcoal never showed reliable results and mostly resulted in "no peaks found", as the charcoal was always isolated with the bacteria from the positive cultures.

Spiked aerobe cultures with reduced incubation times

To decrease the time-to-result even more, aerobe BacT/ ALERT blood culture bottles were spiked with approximately 1,000, 100, and 10 colony-forming units (CFU) of 
S. aureus (ATCC 25923) or E. coli (ATCC 25922) per bottle (representative of 100, 10 and $1 \mathrm{CFU} / \mathrm{ml}$ blood) and were incubated for $7 \mathrm{~h}$ in order to be able to obtain results within one working day $(8 \mathrm{~h})$. Sepsityper was used in combination with MALDI-TOF MS for bacterial identification from these bottles, as this method proved to be superior in previous experiments. After $7 \mathrm{~h}$ of incubation in the BacT/ALERT 3D system, a 1-ml aliquot was taken from each bottle and processed as described in the Sepsityper manual. The culture bottles were placed back in the BacT/ALERT 3D system to determine the time to positivity for each bottle and to use these bottles as positive controls for the Sepsityper procedure the next day. All experiments were performed three times (independently). Reduced incubation times of $7 \mathrm{~h}$ did not result in a peak pattern; no peaks were detected even after the manual selection of areas on the target plate. The $S$. aureus-spiked bottles were flagged positive after $16 \mathrm{~h}(1,000 \mathrm{CFU}), 17.5 \mathrm{~h}$ (100 CFU) and $19.5 \mathrm{~h}(10 \mathrm{CFU})$ by the BacT/ALERT 3D system, and did show the correct identification $(\geq 1.7 \times<2$ or $>2$ ) of S. aureus with the Sepsityper method. The E. colispiked cultures were flagged positive after $12 \mathrm{~h}$ (1,000 CFU), $13 \mathrm{~h}(100 \mathrm{CFU})$ and $14 \mathrm{~h}(10 \mathrm{CFU})$ by the BacT/ALERT 3D system, and were correctly identified $(>2)$ as containing E. coli by MALDI-TOF MS analysis. As $1 \mathrm{ml}$ might be a low amount of material to analyse after only $7 \mathrm{~h}$ of incubation, it was hypothesised that an increase in the sample volume might lead to the reliable identification of $S$. aureus-spiked aerobe cultures; therefore, the centrifugation/washing method $(4 \mathrm{ml})$ was tested as well as the Sepsityper method, for which $2 \mathrm{ml}$ was used (the chemicals from the kit were increased in the same ratio). Again, for both methods, no reliable identification was found as a result, but the positive bottles did show the reliable identification of $S$. aureus the next day $(\geq 1.7 \times<2$ or $>2$ ). As a last effort, a new protocol was investigated which was published by Moussaoui et al. [14] and describes the use of serum separation tubes (Becton Dickinson). This protocol was optimised for $3.5 \mathrm{ml}$ of culture material and combined with the saponin pre-treatment step as described by Lupetti et al. [20]. The results indicated a non-reliable identification after $7 \mathrm{~h}$ of incubation for $S$. aureus-spiked cultures. The positive bottles did result in reliable identification $(\geq 1.7 \times<2)$ the next day.

\section{Discussion}

In this study, Sepsityper was shown to be the best method for positive aerobe BacT/ALERT blood cultures, as 79/101 cultures were correctly identified (78\%). By using the MolYsis Basic (Molzym) method, 50.5\% of all bottles were correctly identified, and the centrifugation/washing method according to Ferreira et al. [11] resulted in a correct identification rate of $65 \%$ of all cultures. In the current literature, a correct identification rate of between 30 and $90 \%$ is described $[10,12,14,15,17]$, and this is in agreement with the data from this study. Christner et al. [15] and Moussaoui et al. [14] both describe high correct identification rates of approximately $90 \%$, which is higher than the $78 \%$ found in this study. The most important difference is the use of BACTEC cultures (compared to BacT/ALERT cultures) in those studies. In addition, a clear difference was observed between the identification of Gram-negative versus Gram-positive bacteria, 96\% versus $66 \%$, respectively (Sepsityper), which confirms the observations of previous studies $[11,12]$.

Streptococci were found to be problematic pathogens for MALDI-TOF MS identification directly from positive cultures. In three cases, S. pneumoniae had to be detected by LytA real-time PCR, because no growth was observed on agar media, as the bacteria died in the bottles. In these three specific cases, no reliable identification from the positive cultures was possible with all of the tested methods. For the other bottles containing streptococci $(n=9)$, the direct identification from blood culture material resulted mostly in unreliable identification $(<1.7)$ as well, while the identification from agar media was not problematic for the majority ( $7 / 9$ cases). The problems encountered in this study have also been observed by others [12].

In pilot experiments, it was found that anaerobe bottles more frequently resulted in unreliable data. Therefore, in this study, only positive aerobe BacT/ALERT cultures were used. The reason for the better performance of aerobe cultures might be found in the fact that the most commonly detected bacteria grow better in aerobe culture circumstances. This can, subsequently, result in a higher bacterial load in aerobe culture bottles and, subsequently, in better MALDI-TOF MS spectra. Further studies should focus on the usage of anaerobe BacT/ALERT blood cultures for the optimal implementation of MALDI-TOF MS in the clinical microbiology laboratory. In contrast, for BACTEC bottles, several groups have described similar results for both positive aerobe and anaerobe cultures [10, 15]. This observation needs to be properly addressed in the future.

Previously, we showed the possibility of reduced incubation times for blood cultures spiked with staphylococci (1 CFU/ml blood); only $5 \mathrm{~h}$ of pre-incubation was sufficient to detect staphylococci when using real-time PCR as an analytical tool [4]. In this study, a pre-incubation of $7 \mathrm{~h}$ was investigated by using $S$. aureus- and E. coli-spiked BacT/ ALERT bottles for subsequent rapid direct analysis with MALDI-TOF MS. Several approaches were undertaken but these never resulted in reliable results. It has been described that at least $>10^{8} \mathrm{CFU} / \mathrm{ml}$ need to be present in culture material in order to obtain a spectra that resembles that from 
a pure culture, and at $10^{6} \mathrm{CFU} / \mathrm{ml}$, the spectra resembles that of a sterile culture [15]. This can be the explanation for the results obtained in this study. It remains unexplained why unreliable data have been obtained when $4 \mathrm{ml}$ was used after $7 \mathrm{~h}$ of incubation.

In this study, $S$. aureus-containing cultures were never misidentified as CNS and vice versa. Ferroni et al. [16] showed similar results with positive BacT/ALERT bottles concerning staphylococci. This may contribute to the discrimination of contamination versus true infection. Another positive effect of MALDI-TOF MS identification is that CNS are now more frequently identified to the species level. This group of microorganisms is increasingly described in the literature in relation to infection [21, 22], making it clinically relevant to investigate this group of pathogens in more detail.

Positive BacT/ALERT bottles were tested immediately in the morning $(9 \mathrm{am})$. If bottles became positive during the night in the BacT/ALERT 3D system, they remained in the culture machine until $8.30 \mathrm{am}$, and the time to positivity (TTP) was not registered for this study. Bottles were kept at room temperature, when positive, between 8.30 am and 6 $\mathrm{pm}$ (working hours); subsequently, they were stored for 1 week at $35^{\circ} \mathrm{C}$. Bottles that became positive in the afternoon were tested the next day. However, if only Sepsityper is used for the analysis, positive bottles can be collected several times a day; for example, every $2 \mathrm{~h}$, starting at $8.30 \mathrm{am}$, with the last round at $5.00 \mathrm{pm}$.

The results of this study implicate that routine diagnostics can be optimised when positive blood cultures can be processed by Sepsityper followed by MALDI-TOF MS, as schematically represented in Fig. 3b. Pathogen identification can be available within $20 \mathrm{~min}$ (depending on the number of positive cultures that need to be processed), in contrast to the current workflow (approximately 1-2 days; see Fig. 3a). This rapid direct identification of microorganisms from positive cultures can have a major impact in the clinic. Patient-tailored treatment decisions can be made more rapidly, but the final antibiotic therapy can only be installed if antibiotic susceptibility results are available. Although Sepsityper was superior in this study, alternatives to this approach exist and clinical microbiology laboratories
Fig. 3 Schematic representation of the current (a) and possible new diagnostic workflow (b). Every positive culture (anaerobe cultures need further research) will be processed by Sepsityper and analysed by using MALDITOF MS to have optimal speed for the identification of pathogens. Additionally, Gram staining and subculture on agar media will be started for antibiotic susceptibility testing and, when necessary, these agar media can be used for MALDITOF MS when the direct identification from cultures is unsuccessful
A

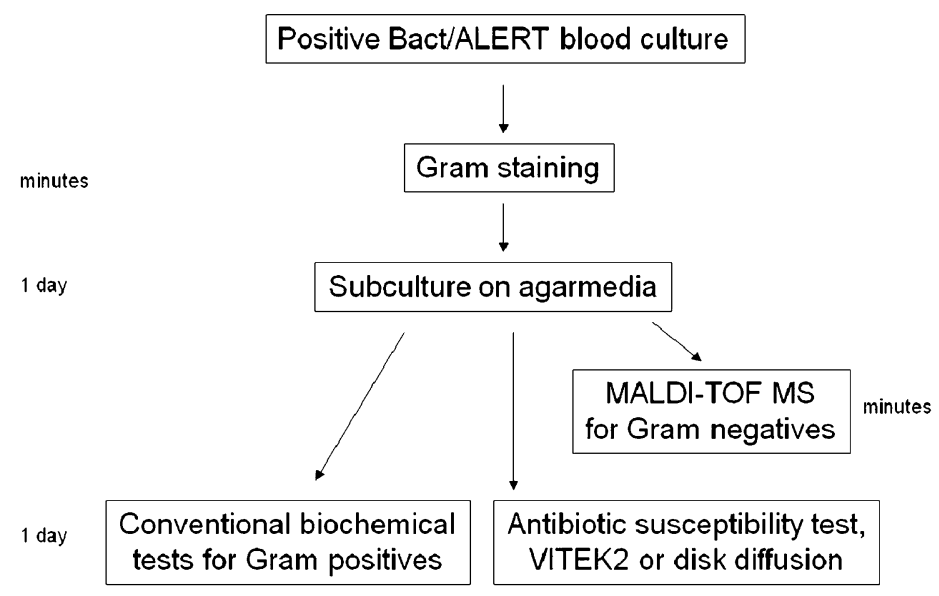

B

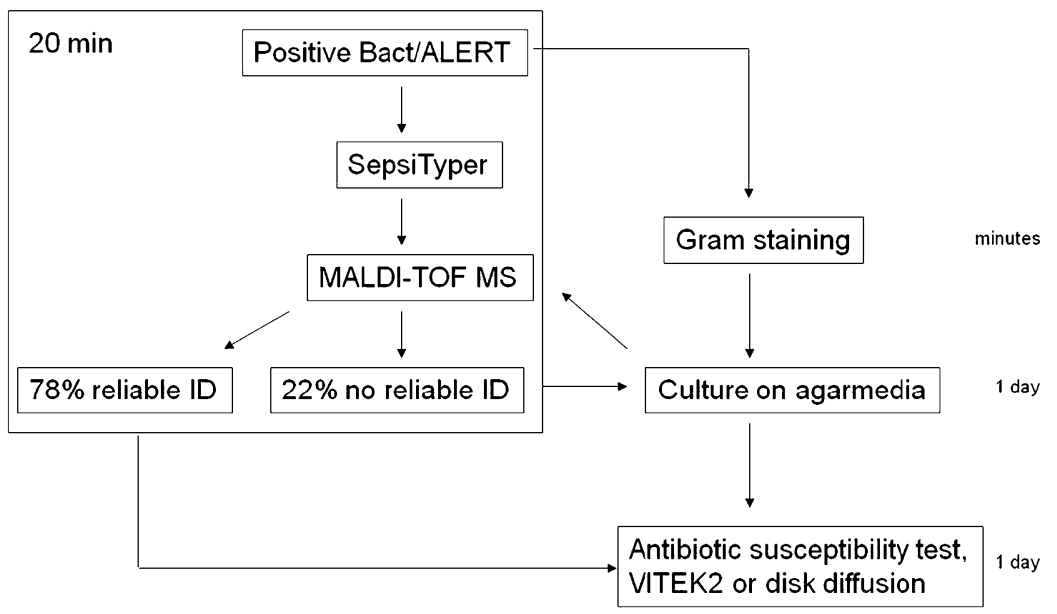


with a lower budget might implement the centrifugation/ washing method for positive cultures containing Gramnegative pathogens and apply the Sepsityper method only for positive cultures containing Gram-positive pathogens. Another alternative might be the usage of the spot subculture agar plates (materials and methods) for cultures which became positive early in the morning and Sepsityper for cultures that became positive later during the day. At the end of the working day, these spot subcultures contain enough bacteria for MALDI-TOF MS analysis via the direct smear method. Unfortunately, it is still not possible to use MALDI-TOF MS for antibiotic susceptibility testing or for the detection of virulence factors. Additional studies are needed for this.

In summary, after the analysis of more than 100 positive aerobe BacT/ALERT cultures with three different processing methods, it can be concluded that Sepsityper is superior for the detection of pathogens directly from positive aerobe BacT/ALERT cultures by MALDI-TOF MS in comparison to processing cultures by MolYsis or the centrifugation/ washing method. Gram-negative pathogens are easier to identify from cultures compared to Gram-positive microorganisms. Reduced incubation $(7 \mathrm{~h})$ of $S$. aureus- and $E$. coli-spiked aerobe BacT/ALERT cultures did not result in reliable identification with all of the methods tested.

Acknowledgements We thank Wouter Bolderdijk for the technical assistance and Lieke Wielders, MSc for her valuable contribution to this manuscript.

Conflict of interest The authors declare that they have no conflict of interest.

Open Access This article is distributed under the terms of the Creative Commons Attribution Noncommercial License which permits any noncommercial use, distribution, and reproduction in any medium, provided the original author(s) and source are credited.

\section{References}

1. Kumar A, Ellis P, Arabi Y, Roberts D, Light B, Parrillo JE, Dodek P, Wood G, Kumar A, Simon D, Peters C, Ahsan M, Chateau D; Cooperative Antimicrobial Therapy of Septic Shock Database Research Group (2009) Initiation of inappropriate antimicrobial therapy results in a fivefold reduction of survival in human septic shock. Chest 136(5):1237-1248

2. Kumar A, Roberts D, Wood KE, Light B, Parrillo JE, Sharma S, Suppes R, Feinstein D, Zanotti S, Taiberg L, Gurka D, Kumar A, Cheang M (2006) Duration of hypotension before initiation of effective antimicrobial therapy is the critical determinant of survival in human septic shock. Crit Care Med 34(6):1589-1596

3. Peters RP, van Agtmael MA, Simoons-Smit AM, Danner SA, Vandenbroucke-Grauls CM, Savelkoul PH (2006) Rapid identification of pathogens in blood cultures with a modified fluorescence in situ hybridization assay. J Clin Microbiol 44(11):4186-4188
4. Loonen AJ, Jansz AR, Kreeftenberg H, Bruggeman CA, Wolffs PF, van den Brule AJ (2011) Acceleration of the direct identification of Staphylococcus aureus versus coagulasenegative staphylococci from blood culture material: a comparison of six bacterial DNA extraction methods. Eur J Clin Microbiol Infect Dis 30(3):337-342

5. Wallet F, Nseir S, Baumann L, Herwegh S, Sendid B, Boulo M, Roussel-Delvallez M, Durocher AV, Courcol RJ (2010) Preliminary clinical study using a multiplex real-time PCR test for the detection of bacterial and fungal DNA directly in blood. Clin Microbiol Infect 16 (6):774-779

6. Wellinghausen N, Kochem AJ, Disqué C, Mühl H, Gebert S, Winter J, Matten J, Sakka SG (2009) Diagnosis of bacteremia in whole blood samples by use of a commercial universal 16S rRNA gene-based PCR and sequence analysis. J Clin Microbiol 47 (9):2759-2765

7. van Veen SQ, Claas EC, Kuijper EJ (2010) High-throughput identification of bacteria and yeast by matrix-assisted laser desorption ionization-time of flight mass spectrometry in conventional medical microbiology laboratories. J Clin Microbiol 48(3):900-907

8. Sogawa K, Watanabe M, Sato K, Segawa S, Ishii C, Miyabe A, Murata S, Saito T, Nomura F (2011) Use of the MALDI BioTyper system with MALDI-TOF mass spectrometry for rapid identification of microorganisms. Anal Bioanal Chem 400(7):1905-1911

9. Bizzini A, Jaton K, Romo D, Bille J, Prod'hom G, Greub G (2011) Matrix-assisted laser desorption ionization-time of flight mass spectrometry as an alternative to 16S rRNA gene sequencing for identification of difficult-to-identify bacterial strains. J Clin Microbiol 49(2):693-696

10. Stevenson LG, Drake SK, Murray PR (2010) Rapid identification of bacteria in positive blood culture broths by matrix-assisted laser desorption ionization-time of flight mass spectrometry. J Clin Microbiol 48(2):444-447

11. Ferreira L, Sánchez-Juanes F, Porras-Guerra I, García-García MI, García-Sánchez JE, González-Buitrago JM, Muñoz-Bellido JL (2011) Microorganisms direct identification from blood culture by matrix-assisted laser desorption/ionization time-of-flight mass spectrometry. Clin Microbiol Infect 17(4):546-551

12. La Scola B, Raoult D (2009) Direct identification of bacteria in positive blood culture bottles by matrix-assisted laser desorption ionisation time-of-flight mass spectrometry. PLoS One 4(11):e8041

13. Prod'hom G, Bizzini A, Durussel C, Bille J, Greub G (2010) Matrix-assisted laser desorption ionization-time of flight mass spectrometry for direct bacterial identification from positive blood culture pellets. J Clin Microbiol 48(4):1481-1483

14. Moussaoui W, Jaulhac B, Hoffmann AM, Ludes B, Kostrzewa M, Riegel P, Prévost G (2010) Matrix-assisted laser desorption ionization time-of-flight mass spectrometry identifies $90 \%$ of bacteria directly from blood culture vials. Clin Microbiol Infect 16 (11):1631-1638

15. Christner M, Rohde H, Wolters M, Sobottka I, Wegscheider K, Aepfelbacher M (2010) Rapid identification of bacteria from positive blood culture bottles by use of matrix-assisted laser desorption-ionization time of flight mass spectrometry fingerprinting. J Clin Microbiol 48(5):1584-1591

16. Ferroni A, Suarez S, Beretti JL, Dauphin B, Bille E, Meyer J, Bougnoux ME, Alanio A, Berche P, Nassif X (2010) Real-time identification of bacteria and Candida species in positive blood culture broths by matrix-assisted laser desorption ionization-time of flight mass spectrometry. J Clin Microbiol 48(5):1542-1548

17. Szabados F, Michels M, Kaase M, Gatermann S (2011) The sensitivity of direct identification from positive BacT/ALERT TM (bioMérieux) blood culture bottles by matrix-assisted laser desorption ionization time-of-flight mass spectrometry is low. Clin Microbiol Infect 17(2):192-195 
18. Hansen WL, Bruggeman CA, Wolffs PF (2009) Evaluation of new preanalysis sample treatment tools and DNA isolation protocols to improve bacterial pathogen detection in whole blood. J Clin Microbiol 47(8):2629-2631

19. McAvin JC, Reilly PA, Roudabush RM, Barnes WJ, Salmen A, Jackson GW, Beninga KK, Astorga A, McCleskey FK, Huff WB, Niemeyer D, Lohman KL (2001) Sensitive and specific method for rapid identification of Streptococcus pneumoniae using realtime fluorescence PCR. J Clin Microbiol 39(10):3446-3451
20. Lupetti A, Barnini S, Castagna B, Capria AL, Nibbering PH (2010) Rapid identification and antimicrobial susceptibility profiling of gram-positive cocci in blood cultures with the Vitek 2 system. Eur J Clin Microbiol Infect Dis 29(1):89-95

21. Piette A, Verschraegen G (2009) Role of coagulase-negative staphylococci in human disease. Vet Microbiol 134(1-2):45-54

22. von Eiff C, Peters G, Heilmann C (2002) Pathogenesis of infections due to coagulase-negative staphylococci. Lancet Infect Dis 2(11):677-685 